\title{
Correlations between Medical Students' Self Assessment of Communication Skills and Patient-Physician Interaction Assessed by Standardized Patients in Clinical Performance Examination
}

\author{
Seieun $\mathrm{Oh}^{1}$, Woo Jeong $\mathrm{Kim}^{2}$ and Min Young Kim ${ }^{3 *}$ \\ ${ }^{1}$ Department of Nursing, College of Health Sciences, Dankook University, Korea \\ ${ }^{2}$ Department of Emergency Medicine, Jeju National University,Korea \\ ${ }^{3 *}$ College of Nursing,Jeju National University, Korea \\ seieun5@dankook.ac.kr,gurum21@jejunu.ac.kr, musemy2@jejunu.ac.kr
}

\begin{abstract}
The purpose of this study was to identify the correlations between medical students' communication skills and patient-physician interaction of clinical performance examination. A total of 36 fourth-year medical students were enrolled in this study and were surveyed concerning communication skills during clinical performance examination. The survey included 20 items in 4major areas: pre-interview (greeting, verifying patient's name, introducing self, expressing interest, and confirming major symptoms), data collection (asking open-ended questions, reflecting, facilitation, clarifying information, and mid-point summarizing), data provision and patient education (finding out what the patient is thinking, responding to the patient's emotions, explaining in a manner easy to understand, checking for the patient's comprehension, taking additional questions), and non-verbal communication (professional appearance, active and respectful listening, appropriate eye contact, appropriate nodding, appropriate silence). 20 items of communication skills were reclassified to 5 patient-physician interaction items, and compared with patient-physician interaction scores of clinical performance examination. There were no correlations between communication skills and patient-physician interaction scores in two examination items (breast pain and acute abdominal pain). This study demonstrated that medical education of communication skills could not be transferred to improvement of patient-physician interaction in clinical skills assessment.
\end{abstract}

Keywords: Communication,Physician-patient relations, Standardized patient

\section{Introduction}

Communication skills are essential to the effective exchange of critical information between medical professionals and their patients. Eliciting salient data from and providing necessary information to patients in an effective and appropriate manner largely depends on communication skills. Teaching patient-centered communication skills can result in a number of benefits. In the short term, such skills are known to improve medical professionals' communication behaviors with patients. In the long term, they can contribute to improved understanding of patients, enhanced patient self-efficacy, and overall improved decision making based on a richer set of information in both quantity and quality. In other words, communication training can improve treatment outcomes, quality of life, and survival rates as well as reducing treatment cost and discrepancies [1]. Unfortunately, a majority of medical students complain of feeling lost and left alone in this regard as they start interviewing their first patients during clinical training. Although their best efforts at communicating effectively continue after graduation, many medical professionals regret the lack of effective communication training during their school years. 
In fact, it is widely known that patients' complaints more frequently concern communication breakdowns than inappropriate care [2].

In the mid-1960s, Barrows and Abrahams at the University of Southern California developed the first Standardized Patients Program, which was soon widely adopted as a training and evaluation tool in the fields of medical student training, faculty professional development, and doctor training. The program spread worldwide when it worked harmoniously with the Objective Structure Clinical Examination (OSCE) developed by a team of researchers led by Professor Harden at the Dundee University medical school in 1975. According to a 1999 survey by the Association of North American Medical Colleges, more than 120 of the 125 total medical colleges were utilizing standardized patients in the training and evaluation of students [3]. In Korea, the Seoul National University medical school first adopted the standardized patients program in 1996. The adoption set in motion a widespread utilization of the Clinical Performance Examination (CPX), which employed standardized patients, by medical schools around the nation. For successful recruitment, education, and training of standardized patients, a medical school consortium was organized, which has been managing and promoting the CPX in medical schools. In response to the growing adoption of CPX by medical schools and increasing demand for a new set of evaluation criteria for medical education, a practical exam was added to the Korean Medical License Examination (KMLE) in 2009. The addition signifies the efforts to diversify evaluation criteria, improve the quality of medical professionals, encourage curriculum that incorporates more rigorous clinical training, and prepare for the new era of the open medical market.

A great deal of effort and investment was required for the National Health Personnel Licensing Examination Board to incorporate a practical exam into the KMLE. As such, a study was conducted to investigate its validity and specific methods of implementation. Subsequent studies were conducted to develop the exam questions for evaluating patientphysician interaction (PPI) and to design the pass/fail criteria, which are currently being used [4]. The KMLE consists of 6 skill sessions and 6 clinical examination sessions. The latter is subsequently composed of medical history taking, physical examination, notetaking and attitude, patient education, PPI, etc. PPI is measured in all questions pertaining to clinical examination. Five of the scoring criteria made available to the public are "effectively elicited information", "actively listened to what the patient had to say", " tried to understand the patient's situation", " explained in a way that the patient could understand", and "tried to forge a positive relationship with the patient".

Han et al. examined the characteristics of PPI domains through an analysis of the CPX evaluation domains by 15 consortium medical schools. In the study, PPI was evaluated through 7 questions concerning relationship building, listening skills, patient encouragement, creating mood, manners, conveying information, and professional attitude, each of which were scored on a 6-point scale (0-5). The results of the study confirmed that PPI, despite the particularities of the 6 cases used in the CPX, critically influenced physicians' abilities to perform clinical tasks. Additionally, PPI scores and the standardized patients' satisfaction levels were found to be closely tied, indicating that PPI greatly affects patient satisfaction [5]. In response to the growing demand for effective communication skills in the field, today's medical and nursing programs are preparing students for specific situations, such as delivering bad news, as well as equipping them with the basic skills required for clinical interviews. Nevertheless, it is not an easy task to reshape students' existing communication patterns to fit the mold of clinical interviews. It is also not clear whether students' awareness of effective communication skills, or even their successful attainment of those skills, actually transfers to performance in a real-life clinical setting. PPI is known to reflect the patient's satisfaction level. As such, a comprehensive evaluation of PPI domains, which would improve our understanding of communication skills' effect on physicians' clinical performance, would be beneficial in our attempt to provide students with necessary communication skills. 


\section{Design and Subjects}

This study examined the communication skill performance rates and CPX PPI scores of 36 medical school seniors measured during the clinical performance assessment period. The students are educated on medical interviews and communication skills prior to the start of clinical training during their third year of medical school. Their first encounter with the CPX happens upon completion of their clinical training. During their senior year, the students receive additional training on communication skills and PPI as well as individualized coaching through CPX role playing. The clinical performance assessment was conducted upon conclusion of all clinical training required during the first semester of the senior year. Communication skill performance rates were self-assessed using a 5point Likert scale. Students received 1 point for failure to perform almost any of the skills and 5 for performing most of the skills. CPX PPI was measured by standardized patients who were trained and managed by the medical college consortium with which the research institute of the present paper is affiliated.

The standardized patients, who were trained on their roles and evaluation methods, had been participating in the CPX of medical students for numerous years. PPI evaluation followed the same scoring method as adopted by the KMLE, which uses a 4-point Likert scale with 1 point given for a poor performance and 4 for an outstanding performance.

\section{Procedure}

Communication skills were measured in 4 major areas: pre-interview, data collection, data provision and patient education, and non-verbal communication, each consisting of 5 sub-categories. For pre-interview, "greeting", "verifying patient's name", "introducing self", "expressing interest", and "confirming major symptoms" were considered. For data collection, "asking open-ended questions", "reflecting","facilitation", "clarifying information", and "mid-point summarizing" were considered. For data provision and patient education, "finding out what the patient is thinking", "responding to the patient's emotions", "explaining in a manner easy to understand", "checking for the patient's comprehension", and "taking additional questions" were considered. As for non-verbal communication skills, "professional appearance", "active and respectful listening", "appropriate eye contact", "appropriate nodding", and "appropriate silence" were considered.

CPX PPI scores were in accordance with the 5 assessment criteria published by the Korea Health Professional National Exam Board. Relevant items included "effectively elicited information", "attentively listened to what patient had to say", "tried to understand patient's situation", "explained in a way that the patient could understand", and "tried to forge a positive relationship with patient".

In order to compare the performance rates (measured in each detailed interview phase) with the CPX PPI scores, the communication skills were reclassified based on the PPI evaluation items, and relevant performance rates were then combined (Figure 1). Of the 20 communication skills, "confirming major symptoms", "asking open-ended questions", "clarifying information", and "mid-point summarizing" were grouped into "effectively elicited information". "Reflecting", "facilitation", "attentive listening", "appropriate eye contact", "appropriate nodding", and "appropriate silence" were grouped into "attentively listened to what the patient had to say". "Finding out what the patient is thinking" and "reacting to the patient's emotions" were regrouped into "tried to understand the patient's situation". "Explaining using simple words", "checking for patient's comprehension", and "taking additional questions" were grouped into "explained in a manner easy to understand". "Greeting", "verifying patient's name", "introducing self", "expressing interest", and "professional appearance" were grouped into "tried to build a positive relationship with the patient". 


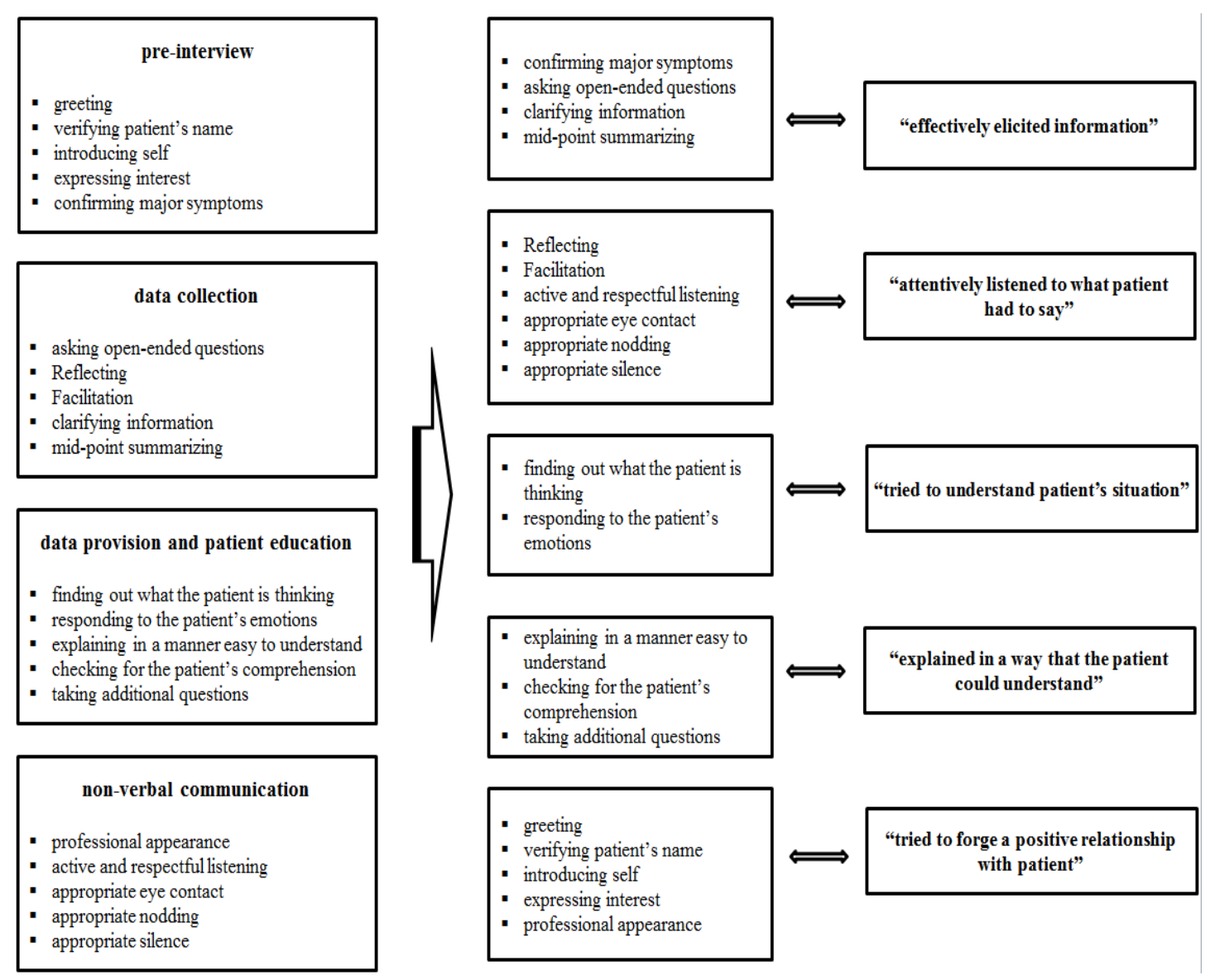

Figure 1. A Total of 20 Communication Skills were Reclassified based on the 5 Items Evaluating Patient-physician Interaction in Clinical Performance Examination

\section{Data Analysis}

The association was examined between the students' self-evaluation scores and the standardized patients' CPX PPI scores pertaining to breast and acute abdominal pain assessment of the CPX items. A Pearson correlation analysis was performed using the SPSS 14.0 (SPSS Inc., Chicago, IL, USA) with a significance level of $p<.05$.

\subsection{Communication Skill Performance Rates}

The highest scores were observed in the area of "confirming major symptoms" at 4.94 \pm 0.23 followed by "greeting" (4.92 \pm 0.28$)$, "introducing self" (4.83 \pm 0.51$)$, "taking additional questions" (4.56 \pm 0.84$)$, "asking open-ended questions" (4.33 \pm 0.79$)$, and "appropriate eye contact" $(4.31 \pm 0.75)$. The communication skill in which students exhibited the poorest performance rates was "finding out what patient is thinking" at 2.36 \pm 1.15 followed by "reacting to patient's emotions" $(2.72 \pm 1.26)$, "checking for comprehension" $(2.75 \pm 1.32)$, and "facilitation" $(2.89 \pm 0.92)$.

\subsection{Analysis of Communication Skills and Clinical Performance Assessment PPI}

The performance rates of 20 communication skills tailored to correspond to the PPI evaluation items and the PPI scores pertaining to the breast pain examination were analyzed. No single skill was found to be significantly associated. The analysis of the PPI scores pertaining to the acute abdominal pain examination also found that no particular skill was meaningfully associated with the scores (Table 1). 
Table 1. Correlations between Scores Modulated from Communication Skills and Patient-physician Interaction Scores of Clinical Performance Examination

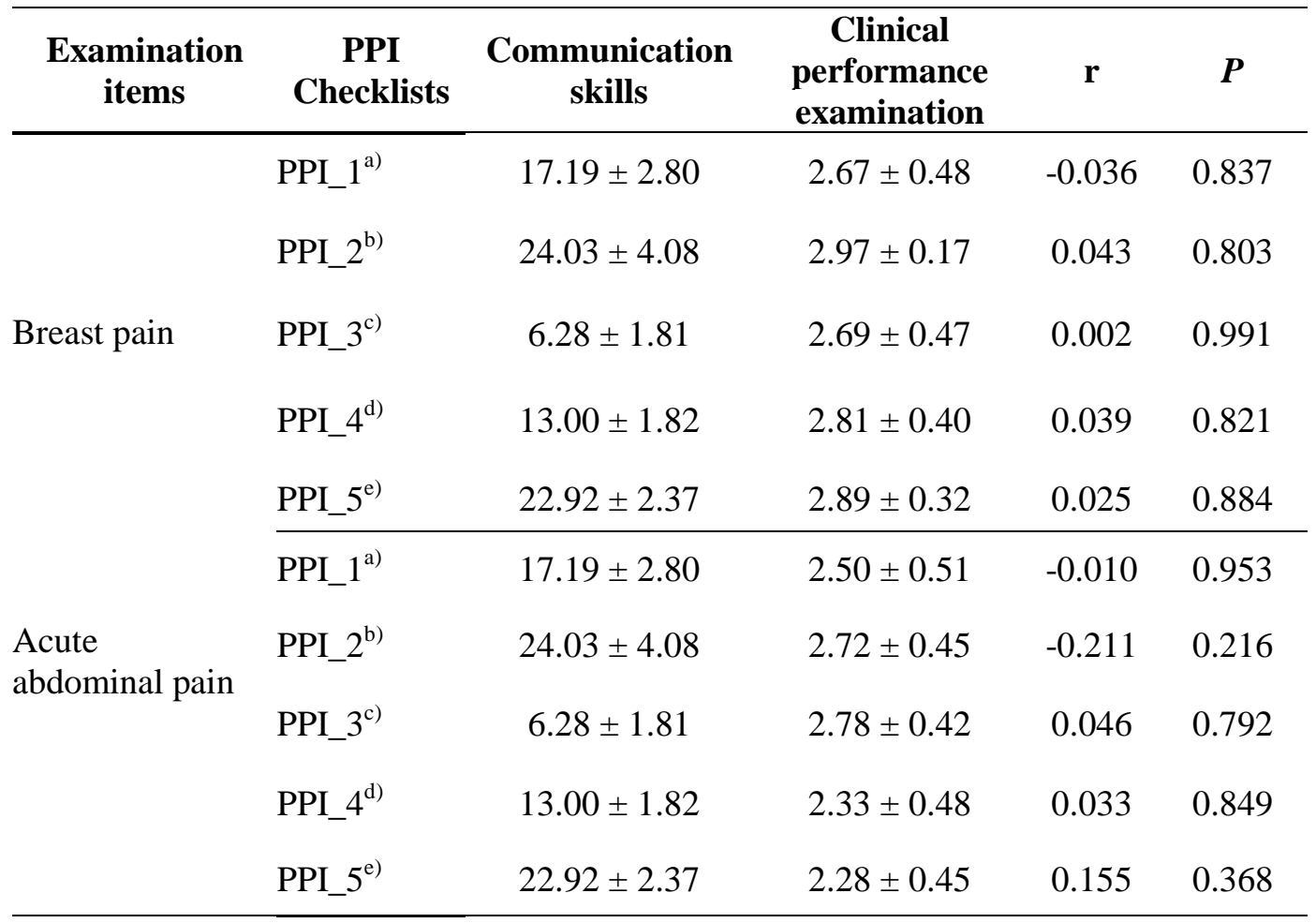

a) evaluated"effectively elicited information"

b) evaluated"attentively listened to what patient had to say"

c) evaluated"tried to understand patient's situation"

d) evaluated "explained in a way that the patient could understand"

e) evaluated "tried to forge a positive relationship with patient"

\section{Discussion}

Ineffective communication with patients can result in inappropriate treatment decisions based on misinformation. It can also negatively affect treatment outcomes by potentially undermining patient's receptiveness to nursing care. Effective communication skills are especially critical in the care of cancer patients or patients in the end stage of other terminal illnesses with whom building a trusting relationship may be challenging using regular communication strategies. Effective communication skills contribute to enhanced patient care by improving our understanding of patients' physical and emotional states and providing us with opportunities to empathize with patients. Basic communication skills begin at the pre-interview phase in the form of greeting, verifying patient, introducing self, and expressing interest in the patients. The subsequent data collection phase requires such skills as appropriate mixing of open-ended and closed questions, empathizing, facilitation, clarifying, mid-point summarizing, and maintaining appropriate silence. During the next phase, in which information is conveyed to the patient, eliciting the patient's thoughts and feelings, reacting to the patient's emotions, explaining in simple terms, checking for comprehension, and taking additional questions are some of 
the communication skills required of physicians. As for non-verbal skills, professional appearance, attentive listening attitude, appropriate eye contact, and appropriate nodding are considered [6].

Education and evaluation of communication skills vary depending on the nation or educational institute. A British national medical school survey identified the OSCE, which takes place an average of 2.4 times per year, as the most widely used tool for evaluating communication skills[7]. The study participants are evaluated twice per year at an 8-month interval. In fact, most of the students receive additional assessment by outside agencies, a fact that brings the figure close to that of the British survey.

Formal communication skill training has been received positively for the most part. A study by Gysels et al. analyzed a total of 47 published studies to investigate the effects of communication training on the treatment of cancer patients. The results indicated that the training improved basic communication skills in the participants and that a positive attitude and belief were required in order to successfully put the learned skills into practice [8]. Kim et al. conducted a study with medical students in their third year of school to examine 10 types of communication skills, which included "initiating interview","active and attentive listening", "expressing empathy", "systematic interview", "understanding the patient's point of view", "explaining in a manner easy to understand", "building relationship with the patient", "non-verbal communication",and "being considerate before and during clinical examination". According to the results, the participants who received 1 week of practical communication skill training scored significantly higher than their counterparts who received 1 week of theoretical training in the particular areas of "initiating interview", "expressing empathy", and "being considerate before and during the clinical examination". Additionally, "initiating interview", "understanding the patient's point of view", and "being considerate before and during clinical examination" tend to improve as the length of training increased. Such training effects are sustained over a long period of time [9]. In a longitudinal study by Park et al., medical students' communication skills were evaluated by the same set of standardized patients during their years in school and after they had become residents. The results showed significant improvements in the area of "explaining in a manner that is easy to understand" [10].

However, the effectiveness of communication training appears to vary greatly according to the particular situation. Additionally, some question the transferability of learned skills to a real-life setting. A study by Jang et al. examined the association between students' academic performance and empathetic communication skills. According to the study results, male students exhibited higher scores in the areas of "interviewing attitude", "willingness to build trust","active and attentive listening", and "willingness to help" [11]. The association between communication skills and academic performance varied depending on the questions. On the other hand, according to a study conducted with intensive care unit physicians and nurse practitioners to investigate the association between communication training and patient assessment, communication training not only failed to improve the quality of communication compared with general training but also intensified patient's depression. Such a finding certainly raises doubts regarding the transferability of learned communication skills to an actual clinical setting [12]. Patient's age and educational attainment also appear to influence the outcomes of communication assessments. A study by Abadel and Hattab reported that, in general, patients over 45 years of age, and those with low educational attainment tend to give physician's communication skills more credit than their younger and better-educated counterparts [13].

In the present study, the participants generally scored highly in "confirming major symptoms", "greeting", "introducing self", "taking additional questions", "asking openended questions", and "making appropriate eye contact". It is thought that the high performance rates may be due to the fact that these communication skills are basic 
communication skills, which are frequently practiced in ordinary settings and effectively internalized. On the other hand, the communication skills with low scores included "finding out what the patient is thinking", "reacting to the patient's emotions", "checking for patient comprehension", and "facilitation". "Finding out what the patient is thinking" was the communication skill on which the participants scored the poorest. In fact, they scored less than half the points on it as were scored on skills such as "confirming major symptoms", "greeting", and "introducing self". These skills require practice for sufficient internalization, as they are not typically utilized in everyday conversations. Nevertheless, their role in successful patient interview is critically important. As such, greater attention and emphasis should be given to the attainment of these skills during communication training.

Generally, patient interview education, including communication training, is provided to students through separate and individual skills training, which are organized in the order of a typical interview process or by concepts. However, in a real-life interview setting, the communication skills may need to be utilized in a random order or even extrapolated. In particular, in a PPI assessment centered on communication skills, evaluation is performed in terms of a context-related, comprehensive interpretation of individual items. In the present study, in which 20 communication skills classified to correspond to the PPI evaluation items were compared with the CPX PPI item scores pertaining to breast and acute abdominal pain examination, no significant correlation was found between the skills and the PPI scores. Such results indicate that the students' awareness of the skills as well as their successful execution in a mock setting may not necessarily translate into improved patient satisfaction in a real interview setting. This finding compels us to further consider how to effectively educate and train our students in communication skills required to reap meaningful benefits during clinical training [14].

The present study has several limitations. First, the medical students participating in the study were recruited from a single medical school, and the sample was relatively small, limiting generalization of the findings. Second, 20 skills were selected as major communication skills to be examined in the study. However, the list does not include all existing communication skills, which means that some skill performance rates were not verified despite the influence on the PPI evaluation. Third, the 20 skills were classified into 5 PPI items. Since PPI is a comprehensive assessment, some of the skills may be classified under multiple items, which is not taken into account in the present study.

The present study found that the students' communication skill performance rates varied and that no correlation was present between the performance rates and PPI evaluation. Communication skills can be a powerful treatment aid, which can contribute to the attainment of target health level. They are necessary skills required to influence others, but simply feeding the content to students is not an effective way of educating them. Some have suggested a more systematic educational program that incorporates problem-solving processes [15]. Further research in this area will be beneficial to ensure successful patient interviews conducted with appropriate communication skills.

\section{References}

[1] W. Levinson, C. S. Lesser and R. M. Epstein, "Developing physician communication skills for patientcentered care", Health Affairs, vol. 29, no. 7, (2010), pp. 1310-1317.

[2] M. Kraft and G. Neitzke, "Communication in medical education: students' demands", Medicine, Health Care and Philosophy, vol. 3, (2000), pp. 185-190.

[3] B. G. Lee, "Recent world trend in performance-based assessments and application of the standardized patient program in korean medical education", Korean JMed Educ., vol. 12, no. 2, (2000), pp. 377-392.

[4] J. H. Kim, "The effects and challenges of clinical skills assessment in the korean medical license examination", Korean Medical Education Review, vol. 15, no. 3, (2013), pp. 136-143.

[5] J. J. Han, M. J. Lee and H. J. Im, "Comprehension of patient-physician interaction through analysis of relationships between domains in clinical performance examination", Korean JMed Educ., vol. 22, no. 3, (2010), pp. 177-184. 
[6] M. Lloyd and R. Bor, Communication skills for medicine.3rd edition, Churchill Livingstone Elsevier: London, (2009).

[7] A. Laidlaw, H. Salisbury, E. M. Doherty and C. Wiskin, "National survey of clinical communication assessment in medical education in the United Kingdom (UK)", BMC Med Educ, vol. 14, (2014), p. 10

[8] M. Gysels, A. Richardson and I. J. Higginson, "Communication training for health professionals who care for patients with cancer: a systematic review of effectiveness", Support Care Cancer, vol. 12, (2004), pp. 692-700.

[9] Y. H. Kim, J. H. Yang, S. Y. Ahn, S. Y. Song and H. R. Roh, "Communication skills improvement of medical students according to length and methods of preclinic training”, Korean JMed Educ., vol. 21, no. 1, (2009), pp. 3-16.

[10] W. B. Park, S. R. Phyo, E. Y. Jang, S. H. Kang, S. J. Myung, H. Y. Shin and J. S. Shin, "Correlations of communication and interpersonal skills between medical students and residents", Korean JMed Educ., vol. 22, no. 4, (2010), pp. 269-274.

[11] S. S. Jang, J. H. Seo, G. J. Cho, S. C. Hong and H. O. Woo, "Correlation of communication skills for emotional empathy and academic achievement on clinical performance examinations", Korean JMed Educ., vol. 22, no. 2, (2010), pp. 121-130.

[12] J. R. Curtis, A. L. Back, D. W. Ford, L. Downey, S. E. Shannon, A. Z. Doorenbos and R. A. Engelberg, "Effect of communication skills training for residents and nurse practitioners on quality of communication with patients with serious illness", JAMA, vol. 310, no. 21, (2013), pp. 2271-2281.

[13] F. T. Abadel and A. S. Hattab, "Patients' assessment of professionalism and communication skills of medical graduates", BMC Med Educ., vol. 14, (2014), p. 28

[14] W. J. Kim and M. Y. Kim, "Correlations between communication skills and patient-physician interaction of clinical performance examination", ASTL (advanced science and technology letters), Current Research on Healthcare and Nursing, vol.72, (2014), pp.40-42.

[15] B. Üstün, "Communication skills training as part of a problem-based learning curriculum", J Nurs Educ., vol. 45, no. 10, (2006), pp. 421-424. 